\title{
La Revue de médecine périnatale a un an
}

\author{
P. Lequien \\ C) Springer-Verlag France 2010
}

La Revue de médecine périnatale a franchi le cap de la première année et le moment est venu d'un premier bilan. Il convient au préalable d'adresser des remerciements aux éditions Springer-Verlag France qui ont pris le risque de cette aventure, à Nathalie Huilleret, directrice éditoriale, et à l'équipe dédiée plus spécifiquement à la Revue, Méline Berthelot et Marie-Elia Come-Garry. Leur aide et leur soutien n'ont à aucun moment fait défaut. À ceux qui ont accepté de constituer le comité de rédaction : ils n'ont été économes ni de leur temps ni de leur énergie... ni du recours à leurs réseaux relationnels. Ce sont essentiellement les liens d'amitié qui ont permis de mobiliser les premiers auteurs. Sous le régime de la tyrannie de l'impact factor, la contribution à une revue naissante témoigne d'une incontestable générosité et d'une confiance dans son avenir. Au moment de l'évaluation de leurs travaux par les instances académiques, le travail auquel ils ont consenti ne sera pas « rentable », aussi longtemps que la revue n'est pas répertoriée dans les bases de données internationales. C'est cet objectif qui est visé lorsqu'est imposée la contrainte de la traduction en anglais du titre et du résumé. Aux reviewers, enfin, seuls ceux qui n'ont jamais été sollicités pour assumer ce pensum ignorent combien il réclame de temps, d'attention et, à la fois, d'exigence et de tolérance.

Les objectifs qui avaient été définis par la société, dont elle est l'organe, ont-ils été atteints ? La réponse est oui, en ce qui concerne la pluridisciplinarité, même si la contribution obstétricale est pour l'instant modeste : mais cette discipline comporte tant de publications spécialisées que ce constat n'est pas surprenant. Cette contribution est néanmoins indispensable : nul doute qu'elle se développera dans les mois à venir. C'est l'occasion de rappeler que la revue offre l'opportunité de diffuser les informations et les messages portés par les travaux publiés en langue anglaise. Ils présentent, dans leur grande majorité, un intérêt qui concerne l'ensemble de la collectivité périnatale, dont une faible minorité seulement lit régulièrement les journaux anglo-saxons. Qu'il s'agisse d'une « re-mouture » en français de l'article original, d'une synthèse ou de toute autre forme, ils contribuent à l'information et à la formation du lectorat francophone.

L'irruption - le terme est choisi à dessein — des usagers est un fait majeur. La Revue peut s'enorgueillir d'être un des premiers, sinon le premier organe professionnel francophone à leur ouvrir ses colonnes. Son mérite doit être tempéré si on se souvient que leur existence a été officiellement reconnue et leur place titularisée dans les décrets sur la périnatalité, il y a maintenant plus de dix ans. Leurs contributions n'ont pas été sans susciter parfois de vifs débats au sein du comité de rédaction : elles sont de fait souvent dérangeantes. Les pessimistes s'y résignent, comme ils se résignent, mal parfois, à la fin du paternalisme médical au terme duquel ce sont les professionnels qui savent ce qui est bon pour le patient. Les autres ont cessé de voir en eux sinon des adversaires, du moins des interlocuteurs malveillants. Si cette conception a pu être parfois justifiée, elle l'est de moins en moins. Les usagers d'aujourd'hui alimentent leur réflexion et leurs propositions par une lecture rigoureuse de la littérature médicale, dont ils ne manquent pas d'identifier les fréquentes incohérences et contradictions. Il leur est alors reproché de ne pas disposer de tous les éléments nécessaires à la pertinence d'une lecture critique. C'est du dialogue que naîtront les idées constructives qui permettront de réaliser les objectifs de la santé tels qu'ils sont définis par l'OMS. C'est la vocation de la rubrique « courrier des lecteurs » que d'en être le support, en relayant les opinions divergentes. La qualité et l'intérêt de la Revue appartiennent en définitive aussi aux lecteurs et, à ce titre, leurs contributions sont bienvenues.

La parution de ce numéro au mois de mars est bien tardive pour adresser des vœux. Il n'est, par contre, pas d'époque pour en formuler, un au moins : ce sera celui de la vitalité de l'exercice qui nous rassemble.

P. Lequien

e-mail : pierre.lequien@aliceadsl.fr 